\title{
Protein profiling of Guillain-Barrè syndrome cerebrospinal fluid by two-dimensional electrophoresis and mass spectrometry
}

\author{
Simona D’Aguanno a,b,1, Diego Franciottac ${ }^{\mathrm{c}, 1}$, Santina Lupisella ${ }^{\mathrm{a}, \mathrm{b}, 1}{ }^{\text {, Alessandra Barassi }}{ }^{\mathrm{d}}$, \\ Damiana Pieragostino $^{\mathrm{f}, \mathrm{g}}$, Alessandra Lugaresi ${ }^{\mathrm{f}, \mathrm{g}}$, Diego Centonze $^{\mathrm{a}, \mathrm{h}}$, \\ Gianlodovico Melzi D’Eril ${ }^{\mathrm{d}}$, Sergio Bernardini ${ }^{\mathrm{b}, \mathrm{e}}$, Giorgio Federici ${ }^{\mathrm{b}, \mathrm{e}}$, Andrea Urbani ${ }^{\mathrm{a}, \mathrm{b}, *}$ \\ a Centro Europeo Ricerca sul Cervello, IRCCS-Fondazione Santa Lucia, Rome, Italy \\ b Dipartimento di Medicina Interna, Università di Roma Tor Vergata, Rome, Italy \\ ${ }^{\mathrm{c}}$ Foundation Neurological Institute C. Mondino, IRCCS, Pavia, Italy \\ d Dipartimento di Medicina, Chirurgia e Odontoiatria, Università degli Studi di Milano, Italy \\ e Laboratori di Ricerca, Ospedale Pediatrico Bambin Gesù-IRCCS, Rome, Italy \\ ${ }^{\mathrm{f}}$ Centro Studi sull'Invecchiamento (Ce.S.I.), Chieti, Italy \\ g Università “G. D’Annunzio" di Chieti-Pescara, Italy \\ h Dipartimento di Neuroscienze, Università di Roma “Tor Vergata”, Italy
}

\section{A R T I C L E I N F O}

\section{Article history:}

Received 30 July 2010

Accepted 20 August 2010

\section{Keywords:}

Guillain-Barré syndrome

CSF

Proteomics

Biomarkers

Oxidative damage

Protein carbonylation

\begin{abstract}
A B S T R A C T
Protein profiling of cerebrospinal fluid in Guillain-Barrè syndrome (GBS), an acute and immune-mediated disease affecting the peripheral nervous system, was performed by two-dimensional electrophoresis. Significant modulated spots in GBS patients $v$ s. control groups (a group of multiple sclerosis patients and one of healthy donors) underwent MALDI-TOF/TOF investigation. Inflammation-related proteins, such as vitamin D-binding protein, beta-2 glycoprotein $\mathrm{I}(\mathrm{ApoH})$, and a complement component $\mathrm{C} 3$ isoform were up-regulated in GBS, whereas transthyretin (the monomer and the dimer forms), apolipoprotein E, albumin and five of its fragments were down-regulated. Then, we used an isoelectric-focusingdinitrophenylhydrazine-based technique to analyse the extent of carbonylation and, as a result, of oxidative damage of GBS CSF proteome. We observed a major sensitivity to carbonylation for albumin and alpha-glycoprotein in inflammation and a selective increase of reactivity for a glycosylated Fab from an IgM globulin in GBS CSF. Our results add new proteins to candidate CSF features of GBS, and suggest that oxidative stress could contribute to the immunopathological mechanisms in this syndrome.
\end{abstract}

(C) 2010 Elsevier Ireland Ltd. All rights reserved.
Guillain-Barré syndrome (GBS) is a presumed autoimmune disorder characterized by an inflammatory process that can target myelin sheaths and axons of the peripheral nervous system. The correlated clinical spectrum comprises an acute inflammatory demyelinating polyradiculoneuropathy, which accounts for nearly 95\% of the cases in Europe and North America, and the less frequent acute axonal motor disorder, and acute sensory and motor axonal neuropathy [16]. Clinically, GBS is characterised by acute/subacute ascending symmetrical paresis with areflexia and mild sensory deficits. A minority of patients die notwithstanding prompt intensive care, or show permanent neurological sequelae [17]. The disease is relatively rare (incidence, $1-2$ cases per $10^{5}$ [15]), and affects equally both genders usually at 30-50 age range.

\footnotetext{
* Corresponding author at: University of Rome "Tor Vergata", Dept. of Internal Medicine, Via Montpellier 1, 00133 Rome, Italy. Tel.: +39 06501703215.

E-mail address: andrea.urbani@uniroma2.it (A. Urbani).

1 These authors have equally contributed to this study.
}

The cause of GBS is unknown, but some cases follow a minor infection, usually respiratory or gastrointestinal, and therefore molecular mimicry between microbial antigens and the nerve host tissue might underlie the pathogenesis of these cases [1]. Among microbes, a special role for Campylobacter jejuni has been proposed, given the high resemblance between some of its parietal glycolipids and human peripheral myelin [31]. Diagnostic tests include neurophysiological studies and cerebrospinal fluid (CSF) analysis, which shows a typical inflammatory pattern characterised by the combination of high CSF albumin concentration and normal CSF cell count [17].

Using 2D-electrophoresis (2DE) and MALDI-TOF/TOF techniques, we aimed to investigate the CSF proteome in GBS and, as a result, to characterise the underlying immune-mediated mechanisms, and to identify potential GBS-related biomarkers. Moreover, we analysed the post-translational carbonylation of CSF proteins to evaluate the impact of oxidative stress on such immune-mediated mechanisms.

CSF samples were collected by lumbar puncture from 6 patients with GBS, diagnosed in accordance with Asbury and Cornblath diag- 
Table 1

Clinico-demografic characteristic of patients with Guillain-Barrè syndrome.

\begin{tabular}{|c|c|c|c|c|c|c|c|}
\hline Patient & Male, 1 ; female, 0 & Age at onset (years) & Disease duration (days) & CSF alb (mg/dL) & Serum alb $(\mathrm{mg} / \mathrm{dL})$ & alb \% transfer & CSF (cells $/ \mu \mathrm{L})$ \\
\hline I & 1 & 59 & 4 & 49 & 4049 & 1.2 & $<2$ \\
\hline II & 0 & 15 & 5 & 84 & 4155 & 2.0 & 3 \\
\hline III & 1 & 65 & 25 & 42 & 3660 & 1.1 & $<2$ \\
\hline IV & 0 & 37 & 4 & 81 & 4617 & 1.8 & 2 \\
\hline $\mathrm{V}$ & 0 & 67 & 15 & 96 & 3621 & 2.7 & $<2$ \\
\hline VI & 1 & 43 & 20 & 44 & 4607 & 1.0 & $<2$ \\
\hline Median & & 51 & 10 & 65 & 4102 & 1.5 & $<2$ \\
\hline SE & & 8.2 & 3.7 & 9.7 & 178 & 0.2 & n.a. \\
\hline \multicolumn{4}{|c|}{ Reference ranges } & $10-30$ & $3700-5100$ & $<0.7$ & $<2$ \\
\hline
\end{tabular}

CSF, cerebrospinal fluid; alb, albumin; n.a., not applicable.

nostic criteria [2], from 5 patients affected by multiple sclerosis (MS) as inflammatory controls and from 5 patients who underwent spinal anaesthesia for non-inflammatory orthopaedic pathologies, and whose CSF routine analysis resulted to be normal. Lumbar puncture was performed for diagnostic purposes in GBS patients, whose clinico-demographic characteristics are shown in Table 1. The power setting pre-established from the study according to the sample is definitely under $80 \%$. More details about samples collection and preparation for 2DE, statistical analysis and mass spectrometry analysis are reported in Supplementary files.
The data on blood-brain/CSF barrier permeability, which derived from the calculation of albumin quotients, showed that GBS patients formed into a rather homogeneous group, as they shared a rather similar amount of protein leakage from plasma to CSF (Table 1).

Samples from each subject were analysed individually in replicated experiments after separation of the CSF proteins by 2DE on $18 \mathrm{~cm}$ non-linear 3-10 $\mathrm{pH}$ range 1 st dimension and 2nd dimension on 9-16\% gradient SDS-PAGE (Fig. 1). After silver staining, protein expression profile of each subject was analyzed by PD-Quest soft-
(A)

CTRL

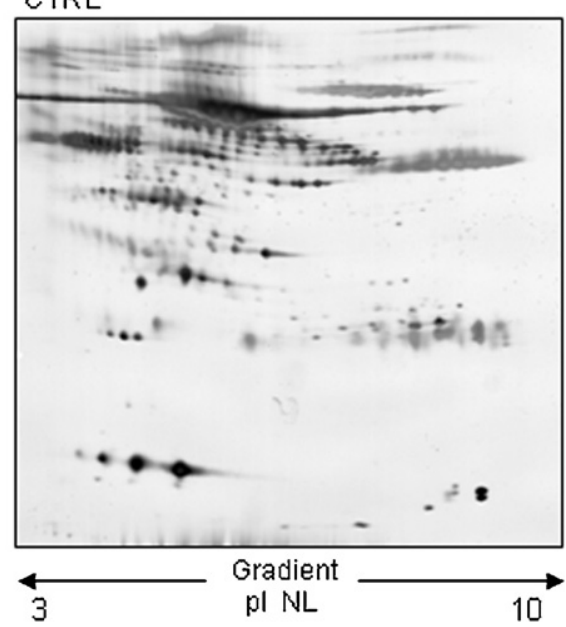

(C)

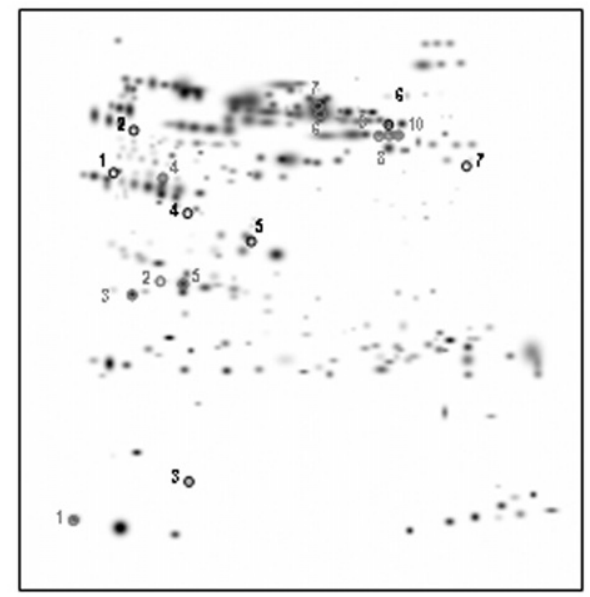

MASTER GEL
(B)

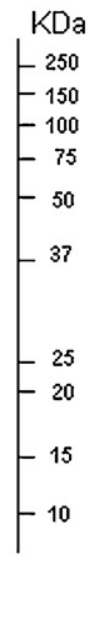

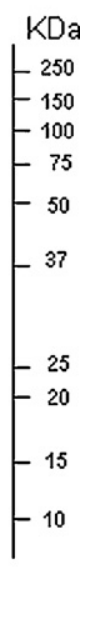

(D)

MS

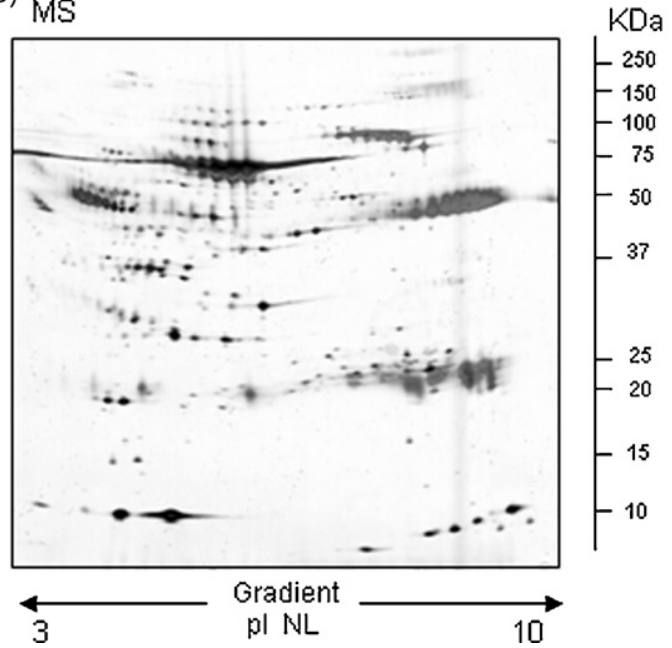

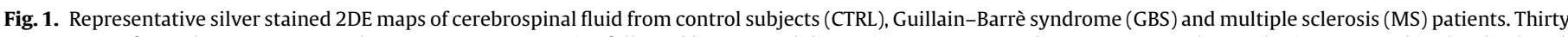

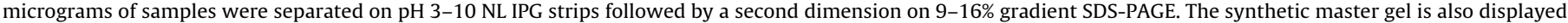

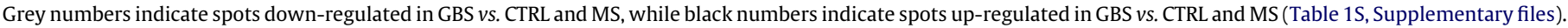


ware. Spots with volume values $>500 \mathrm{ppm}$, showing a significant differential distribution according to the Mann-Whitney test with a ratio above 2 were isolated by excision from the 2DE gels (Fig. 2A and $B$ ), analyzed and identified by Peptide Mass-finger printing and by peptide sequencing (Table $1 \mathrm{~S} \mathrm{~A}$ and B, Supplementary files). A total of 17 differentially expressed spots among the $300 \pm 30$ visualized by silver staining were identified with this screening. Three spots were found to be more represented in GBS: a polypeptide chain belonging to gene product of apolipoprotein A-IV, vitamin D-binding protein and a specific isoform of albumin.

Two spots corresponding to different haptoglobin isoforms, a specific protein spot of beta-2 glycoprotein $\mathrm{I}(\mathrm{ApoH})$, and a spot identified as complement component $\mathrm{C} 3$ fragment were present only in GBS protein maps. The complement component C3 fragment spot was not visible on control maps, although this protein is physiologically present in serum at high concentration, the specific protein isoform associate with these maps seems to be a specific feature of GBS samples. Although the spot values of haptoglobin and complement component $\mathrm{C} 3$ were lower than 500 ppm, we decided to pick them since they were particularly well-defined and reproducible in GBS maps. Ten spots were down-regulated in GBS. Among them, we identified five different additional albumin fragments with an apparent SDS-PAGE molecular mass ranging from 45 to $54 \mathrm{kDa}$ (Table $1 \mathrm{~S} \mathrm{~B}$, Supplementary files).

Moreover three protein spots corresponding to transthyretin (the monomer and the dimer forms) and a spot identified as apolipoprotein E were significantly decreased in GBS.

The extent of protein carbonylation was evaluated in pooled CSF samples from GBS patients, MS patients and non-inflammatory controls by 2DE-Western blot immunoassay (oxyblot, see Supplementary files for more information) (Fig. 3). The subsequent statistical analysis revealed 8 significant differentially-modified spots among GBS and other groups. Corresponding spots in $7 \times 10 \mathrm{~cm}$ silver stained gels were isolated by excision, analyzed and identified by peptide mass-fingerprinting and peptide sequencing using a MALDI-TOF/TOF instrument (Table 2S; Fig. 4 and Supplementary files).

Spots no. 1 and no. 2 were negative on oxyblot staining of GBS CSF pool. They were identified as transthyretin dimer and albumin fragment, respectively. Spot no. 3, an alpha2-HS glycoprotein isoform, had almost no reactivity in the control pool respect to both GBS and MS and about the same level of reactivity in these last groups. Spots no. 4-8 were more reactive in the GBS vs. control pool. Among them spots no. 4-6, recognized by means of NCBI database as albumin protein isoforms, were more carbonylated in MS respect to GBS. Spots no. 7 and no. 8 were associated with a protein sequence highly homologous with a Fab chain from an IgM globulin. While spot no. 7 had the same trend in GBS and MS interesting the spot no. 8 was specifically more reactive in GBS.

For the present proteomic study on CSF samples from GBS patients, we decided to analyse each sample individually, since this strategy seems to be the best compromise in the search for disease-associated proteins $[9,23]$. A different approach, namely the enrichment of CSF samples for low-concentration brain-specific protein detection, would have required large amounts of samples and their pooling. The approach, which needs pre-analytical procedures aimed to deplete the CSF samples of non-cerebral and
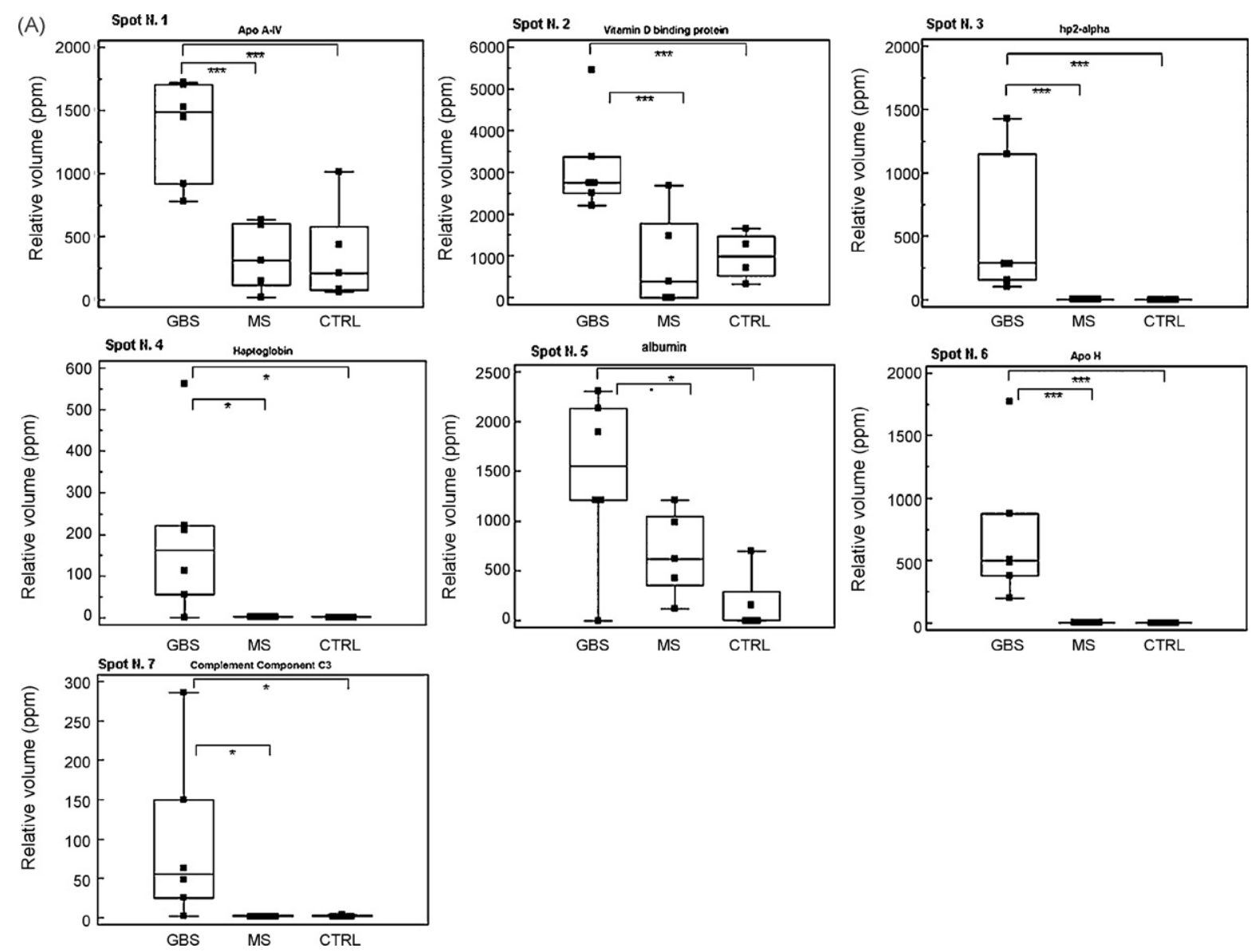

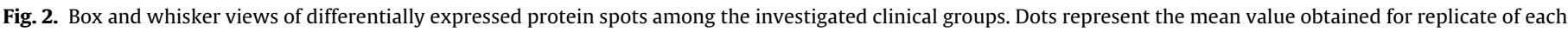

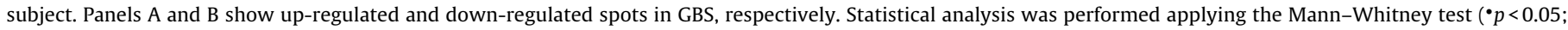
$\left.{ }^{*} p<0.05 ;{ }^{* *} p<0.01 ;{ }^{* * *} p<0.001\right)$. 

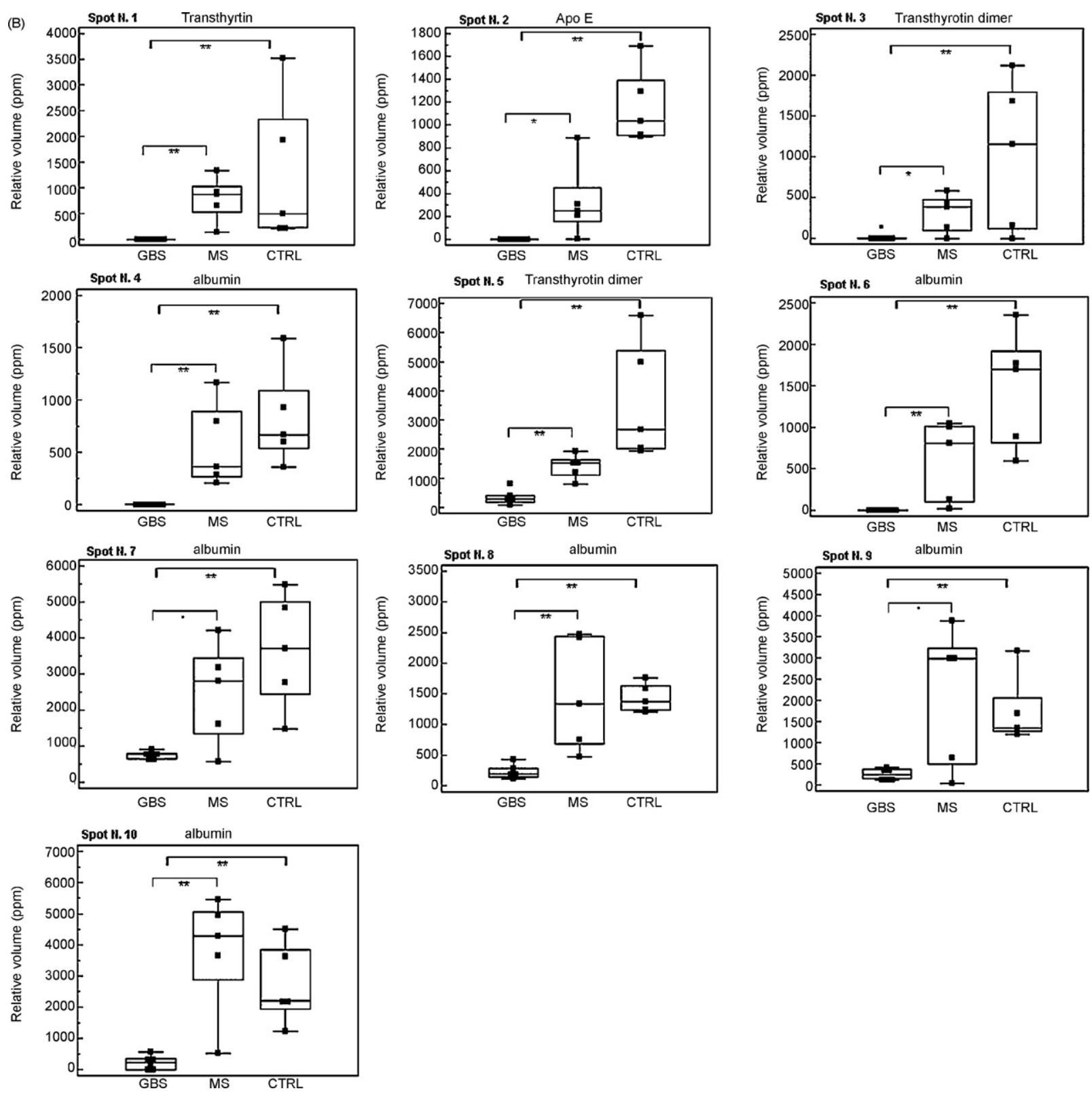

Fig. 2. (Continued)

highly abundant proteins like albumin and immunoglobulins, has some drawbacks, such as the removal of potentially interesting proteins.

Our results, however limited by the sample size which set our statistical power $<80 \%$, provide both confirmatory and novel evidences. Recent proteomic studies showed that apolipoprotein A-IV and haptoglobin were up-regulated, whereas transthyretin and apolipoprotein $\mathrm{E}$ were down-regulated in the CSF of GBS patients $[21,19,33,7]$.

Some proteins, such as transthyretin, apolipoprotein A-IV, vitamin D-binding protein, and haptoglobin, which were differentially regulated in the CSF of our GBS patients vs. controls, have been reported to be expressed in the CSF of patients with very different diseases, which range from neuromyelitis optica, a severe inflammatory CNS disease (transthyretin and vitamin D-binding protein [3]), and viral meningitis or multiple sclerosis (vitamin Dbinding protein [21]), to non-overtly-inflammatory CNS diseases, such as temporal lobe epilepsy (vitamin D-binding protein [32]), lumbar disk herniation (apolipoprotein A-IV and vitamin D-binding protein [23]), Alzheimer disease (transthyretin [26,11]), and amyotrophic lateral sclerosis (transthyretin [25]). As a result, it is likely that the relative-to-total-protein CSF concentration of these proteins is non-specifically influenced by very different neurological pathologies, and therefore it is very unlikely that they can be used as biomarkers in GBS.

Apolipoprotein A-IV is a glycoprotein synthesized by the human intestine, and secreted into the blood, where its levels raises in inflammation [30]. Data on animal models suggest that apolipoprotein A-IV up-regulation in GBS might follow active processes of myelin repair that take place after the acute inflammatory insult [20]. Up-regulation of haptoglobin, beta2-glycoprotein I, and complement component $\mathrm{C} 3$, could thus be explained within the frame of the well-known inflammatory reaction that affects nerve roots in GBS.

Interestingly, we found for the first time that vitamin D-binding protein was up-regulated in the CSF proteome of GBS patients. Similar up-regulations of this multifunctional protein, which acts as a transporter of vitamin D sterols and fatty acids, by activating 

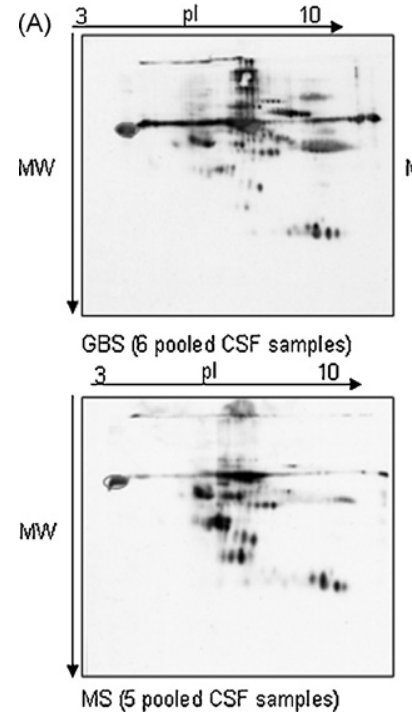

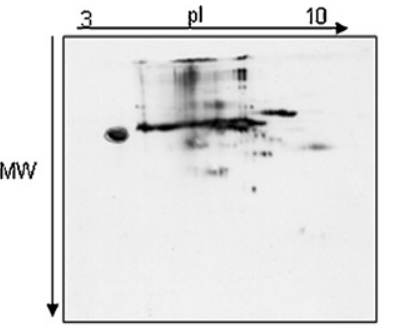

CRTL (5 pooled CSF samples)

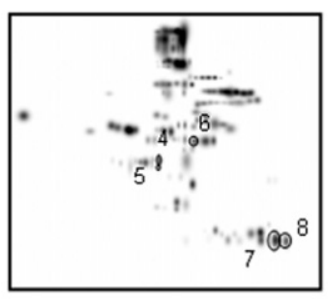

MASTER
(B)

CRTL

GBS

MS

1
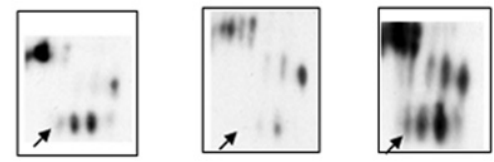

2
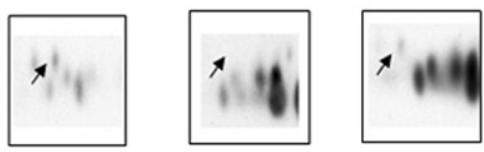

3
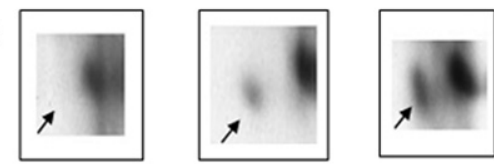

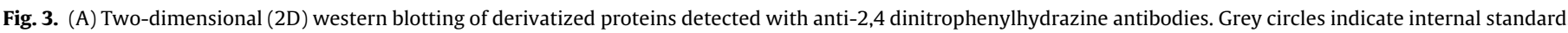

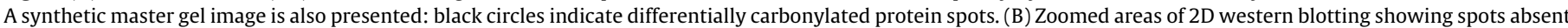
or present in the clinical groups.

macrophage and chemotaxis, and as a scavenger [12], have been reported in many other neurological diseases [23,21,3,32]. Therefore, it is likely that vitamin D-binding protein up-regulation in CSF could represent a non-specific reaction to neurological inflammatory, or even non-inflammatory insults, with possible beneficial functions on post-inflammatory tissue repair.

Transthyretin is a retinol- and thyroxine-binding protein that should play a fundamental role in the brain, a role that is different from that played in the blood, given that its gene is expressed at a high rate in the choroid plexus. The protein is enriched in the CSF, and genetic deficits have been never reported [13]. Transthyretin decreases in the CSF of patients with GBS [19,33,7], as well as in patients with other neurological diseases [21], has been reported. Our data expand these observations, showing a down-regulation of an isoform of transthyretin monomer and of two forms of transthyretin dimer. In contrast with proteomic data, Chiang and Colleagues, using ELISA, reported CSF transthyretin concentrations that were higher in GBS patients than in controls [8]. These findings could be, at least partially, explained by the fact that ELISA measures absolute concentrations of a protein, whereas 2DE yields relative concentrations, namely the ratio with total, and a leakage of plasma into CSF typically characterizes GBS.
Albumin is the most abundant protein in both plasma and CSF, and it is currently used to calculate the degree of blood-CSF barrier damage. Many CSF albumin fragments have been observed in 2DE maps in different pathological conditions $[9,14,5]$. We found an extensive albumin fragmentation in our GBS CSF samples. These fragments were down-regulated in comparison to controls, and therefore the phenomenon should be independent from total albumin concentrations, that are typically increased in GBS [2]. Albumin fragmentation that we observed is likely due to reducing condition of electrophoresis [4]. Another possible explanation of the phenomenon refers to albumin carbonylation, which might make the protein undergo lysosomal degradation. Indeed, our study on CSF protein carbonylation showed evidence of albumin carbonylation in GBS vs. controls, although the degree of carbonylation in controls is higher than in GBS patients.

To evaluate the impact of inflammation-related oxidative stress on GBS CSF proteome, we investigated the distribution of carbonylated proteins. These proteins, being irreparably damaged, are removed by proteolytic degradation, or they accumulate as unfolded proteins [28]. Carbonylation of proteins may provide a direct indication of protein damage caused by oxidative stress [27], as shown in various human diseases $[22,10]$.

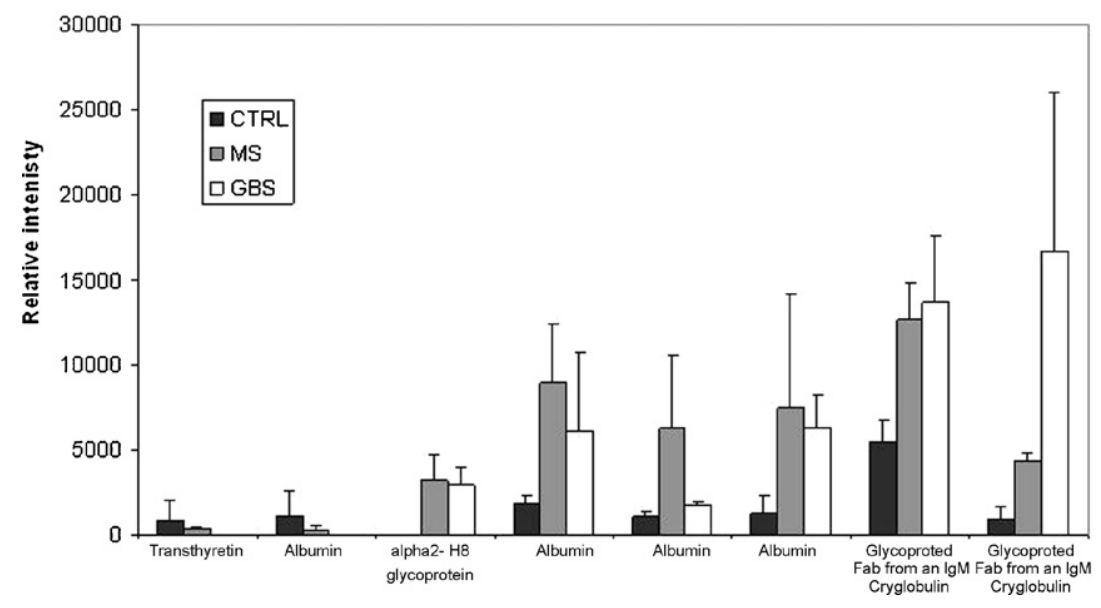

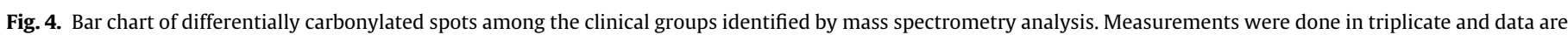
presented as mean + SD. Statistical analysis was performed applying the Student's $T$-test (reported data are significant with $p<0.05$ ) 
We observed eight differentially carbonylated spots, but none corresponded to the albumin fragments or to the transthyretin dimeric forms which have been highlighted in the total protein staining of 2DE maps. Our results indicate that albumin, alpha2glycoprotein, and Fab fragments from an IgM globulin are the major targets of carbonylation.

Albumin can exert an important anti-oxidant function against reactive oxygen species, and our data in GBS parallel those reported in other conditions, such as ageing [6], uremia [18], and hemodialysis [24], that are characterised by oxidative stress. Similarly, alpha2-glycoprotein carbonylation could play a protective, antioxidant role, but no evidence of such role is available in the literature so far. While most of the carbonylated spots seemed to be associated to a general condition of inflammation the spots corresponding to a polypeptide chain that showed extensive sequence homology to a Fab fragment of an IgM cryoglobulin with properties of a natural proteolytic antibody was the most reactive in GBS respect both to MS and non-inflammatory controls. However, we could not demonstrate the cryoglobulin properties of this polypeptide chain in the 2DE. Such properties have been associated with diseases such as rheumatoid arthritis, systemic lupus erythematosus, chronic viral infections, and B-cell malignancies [29], but not with GBS.

In conclusion, the study on carbonylated proteins supports the involvement of oxidative stress in acute inflammatory events that characterise GBS.

Our data indicate that CSF proteome in GBS is characterized mainly by proteins that are non-specifically up-regulated in different neurological diseases, and, at a lesser extent, by proteins involved in inflammation and tissue repair. Carbonylation of some CSF proteins likely follows GBS immunopathological mechanisms. These data will contribute to complete mapping of CSF proteome in GBS. Validation of some interesting data with immunoblotting and ELISA on larger series of GBS patients and appropriate controls will yield possible identification of disease-specific markers.

\section{Acknowledgements}

This manuscript was supported in part by the following grants: Telethon GGP07252, Fondazione Roma 2008, "Rete Nazionale di Proteomica" FIRB RBRN07BMCT.

\section{Appendix A. Supplementary data}

Supplementary data associated with this article can be found, in the online version, at doi:10.1016/j.neulet.2010.08.060.

\section{References}

[1] C.W. Ang, B.C. Jacobs, J.D. Laman, The Guillain-Barré syndrome: a true case of molecular mimicry, Trends Immunol. 25 (2004) 61-66.

[2] A.K. Asbury, D.R. Cornblath, Assessment of current diagnostic criteria for Guillain-Barrè syndrome, Ann. Neurol. 27 (1990) S21-S24.

[3] S. Bai, S. Liu, X. Guo, Z. Qin, B. Wang, X. Li, Y. Qin, Y.H. Liu, Proteome analysis of biomarkers in the cerebrospinal fluid of neuromyelitis optica patients, Mol. Vis. 15 (2009) 1638-1648.

[4] M. Bruschi, L. Musante, G. Candiano, L. Santucci, C. Zennaro, M. Carraro, P. Del Boccio, R. Gusmano, F. Perfumo, A. Urbani, G.M. Ghiggheri, Transitions of serum albumin in patients with glomerulosclerosis 'in vivo' characterization by electrophoretic titration curves, Electrophoresis 27 (2006) 2960-2969.

[5] G. Candiano, L. Musante, M. Bruschi, A. Petretto, L. Santucci, P. Del Boccio, B. Pavone, F. Perfumo, A. Urbani, F. Scolari, G.M. Ghiggheri, Repetitive fragmentation products of albumin and alpha1-antitrypsin in glomerular diseases associated with nephrotic syndrome, J. Am. Soc. Nephrol. 11 (2006)3139-3148.

[6] K. Chandan, C.H. Jana, N. Das, S. Rajindar, R.S. Sohal, Specificity of age-related carbonylation of plasma proteins in the mouse and rat, Arch. Biochem. Biophys. 397 (2002) 433-439.

[7] K.H. Chang, R.K. Lyu, M.Y. Tseng, L.S. Ro, Y.R. Wu, H.S. Chang, W.C. Hsu, H.C. Kuo, C.C. Huang, C.C. Chu, S.Y. Hsieh, C.M. Chen, Elevated haptoglobin level of cere- brospinal fluid in Guillain-Barré syndrome revealed by proteomics analysis, Proteomics Clin. Appl. 1 (2007) 467-475.

[8] H.L. Chiang, R.K. Lyu, M.Y. Tseng, K.H. Chang, H.S. Chang, W.C. Hsu, H.C. Kuo, C.C. Chu, Y.R. Wu, L.S. Ro, C.C. Huang, C.M. Chen, Analyses of transthyretin concentration in the cerebrospinal fluid of patients with Guillain-Barré syndrome and other neurological disorders, Clin. Chim. Acta 405 (2009) 143-147.

[9] S. D’Aguanno, A. Barassi, S. Lupisella, G. Melzi d'Eril, P. Del Boccio, D. Pieragostino, F. Palloti, V. Carelli, M.L. Valentino, R. Liguori, P. Avoni, S. Bernardini, D. Gambi, A. Urbani, G. Federici, Differential cerebro spinal fluid proteome investigation of Leber hereditary optic neuropathy (LHON) and multiple sclerosis, J. Neuroimmunol. 193 (2008) 156-160.

[10] I. Dalle-Donne, R. Rossi, D. Giustarini, A. Milzani, R. Colomco, Protein carbonyl groups as biomarkers of oxidative stress, Clin. Chim. Acta 329 (2003) 23-38.

[11] P. Davidsson, A. Westman-Brinkmalm, C.L. Nilsson, M. Lindbjer, L. Paulson, N Andreasen, M. Sjogren, K. Blennow, Proteome analysis of cerebrospinal fluid proteins in Alzheimer patients, Neuroreport 13 (2002) 611-615.

[12] S.J. Di Martino, G. Trujillo, L.A. McVoy, J. Zhang, R.R. Kew, Upregulation of vitamin D binding protein (Gc-globulin) binding sites during neutrophil activation from a latent reservoir in azurophil granules, Mol. Immunol. 44 (2007) 2370-2377.

[13] W. Duan, M.G. Achen, S.J. Richardson, M.C. Lawrence, R.E. Wettenhall, A Jaworowski, G. Schreiber, Isolation, characterization, cDNA cloning and gene expression of an avian transthyretin. Implications for the evolution of structure and function of transthyretin in vertebrates, Eur. J. Biochem. 200 (1991) 679-687.

[14] E.J. Finehout, Z. Frank, K.H. Lee, Towards two-dimensional electrophoresis mapping of the cerebrospinal fluid proteome from a single individual, Electrophoresis 25 (2004) 2564-7255.

[15] P. Flachenecker, Epidemiology of neuroimmunological diseases, J. Neurol. 253 (2006) 2-8.

[16] R.D. Hadden, D.R. Cornblath, R.A. Hughes, J. Zielasek, H.P. Hartung, K.V. Toyka, A.V.Swan, Electrophysiological classification of Guillain-Barré syndrome: clinical associations and outcome. Plasma exchange/sandoglobulin Guillain-Barré syndrome Trial Group, Ann. Neurol. 44 (1998) 780-788.

[17] H.P. Hartung, J.D. Pollard, G.K. Harvey, K.V. Toyka, Immunopathogenesis and treatment of the Guillain-Barré syndrome, Part I, Muscle Nerve 18 (1995) $137-153$.

[18] J. Himmelfarb, E. McMonagle, Albumin is the major plasma protein target of oxidant stress in uremia, Kidney Int. 60 (2001) 358-363.

[19] T. Jin, L.S. Hu, M. Chang, J. Wu, B. Winblad, J. Zhu, Proteomic identification of potential protein markers in cerebrospinal fluid of GBS patients, Eur. J. Neurol. 14 (2007) 563-568.

[20] C.A. Langner, E.H. Birkenmeier, K.A. Roth, R.T. Bronson, J.I. Gordon, Characterization of the peripheral neuropathy in neonatal and adult mice that are homozygous for the fatty liver dystrophy (fld) mutation, J. Biol. Chem. 266 (1991) 11955-11964.

[21] V. Lehmensiek, S.D. Süssmuth, J. Brettschneider, G. Tauscher, S. Felk, F. Gillardon, H. Tumani, Proteome analysis of cerebrospinal fluid in Guillain-Barré syndrome (GBS), J. Neuroimmunol. 185 (2007) 190-194.

[22] R.L. Lenive, N. Wehr, J.A. Williams, E.R. Stadtman, E. Shacter, Determination of carbonyl groups in oxidized proteins, Methods Mol. Biol. 99 (2000) 15-24.

[23] X.D. Liu, B.F. Zeng, J.G. Xu, H.B. Zhu, O.C. Xia, Proteomic analysis of the cerebrospinal fluid of patients with lumbar disk herniation, Proteomics 6 (2006) 1019-1028.

[24] K. Mera, M. Anraku, K. Kitamura, K. Nakajou, T. Maruyama, K. Tomita, M. Otagiri, Oxidation and carboxy methyl lysine-modification of albumin: possible involvement in the progression of oxidative stress in hemodialysis patients, Hypertens. Res. 28 (2005) 973-980.

[25] S. Ranganathan, E. Williams, P. Ganchev, V. Gopalakrishnan, D. Lacomis, L Urbinelli, K. Newhall, M.E. Cudkowicz, R.H. Brown Jr., R. Bowser, Proteomic profiling of cerebrospinal fluid identifies biomarkers for amyotrophic lateral sclerosis, J. Neurochem. 95 (2005) 1461-1471.

[26] J.M. Serot, D. Christmann, T. Dubost, M. Couturier, Cerebrospinal fluid transthyretin: aging and late onset Alzheimer's disease, J. Neurol. Neurosurg. Psychiatry 63 (1997) 506-508.

[27] E. Shacter, J.A. Williams, M. Lim, R.L. Levine, Differential susceptibility of plasma proteins to oxidative modification: examination by western blot immunoassay, Free Radic. Biol. Med. 17 (1994) 429-437.

[28] E.R. Stadtman, B.S. Barlett, Reactive oxygen-mediated protein oxidation in aging and disease, Drug Metab. Rev. 30 (1998) 225-243.

[29] A.Tedeschi, C. Baratè, E. Minola, E. Morra, Cryoglobulinemia, Blood Rev.4(2007) $183-200$.

[30] G.F. Tu, F. De Jong, J. Apostolopoulos, M. Nagashima, N. Fidge, G. Schreiber, G. Howlett, Effect of acute inflammation on rat apolipoprotein mRNA levels, Inflammation 11 (1987) 241-251.

[31] P.A. van Doorn, L. Ruts, B.C. Jacobs, Clinical features, pathogenesis, and treatment of Guillain-Barré syndrome, Lancet Neurol. 7 (2008) 939-950.

[32] F. Xiao, D. Chen, Y. Lu, Z. Xiao, L.F. Guan, J. Yuan, L. Wang, Z.Q. Xi, X.F. Wang, Proteomic analysis of cerebrospinal fluid from patients with idiopathic temporal lobe epilepsy, Brain Res. 1255 (2009) 180-189.

[33] Y.R. Yang, S.L. Liu, Z.Y. Qin, F.J. Liu, Y.J. Qin, S.M. Bai, Z.Y. Chen, Comparative proteomics analysis of cerebrospinal fluid of patients with Guillain-Barré syndrome, Cell Mol. Neurobiol. 28 (2008) 737-744. 